\title{
Gender Distinctions and Gender Neutrality: Towards a Gender Egalitarian Ethics
}

\author{
Merina Islam*
}

\section{Abstract}

The general mission of feminist philosophy is to correct whatever male biases may exist in the mainstream philosophical traditions. Thus western feminist philosophers investigate and challenge the ways in which western traditions have so long been participating in subordinating women or in rationalizing their subordination. By questioning the gender insensitivity of ethics and philosophy, feminism attempts to reveal various forms of subjugation of women operating through laws, institutions, customs, social theories, and cultural values. Feminism aims at coming up with a better design for society, based on a thorough review and rethinking of gendered positioning of human beings.

\section{Introduction}

The core content of Feminists' critique of traditional or mainstream ethics can be formulated in the form of the following charge: that because of its inherent gender bias ethics has so far been grounding discriminations on gender distinctions or differences. That is to say, by capitalizing sex/gender differences ethics is perpetuating discriminatory practices in the society. As against this, Feminists have adopted the stance that the only possible way

* Assistant Professor, Department of Philosophy, Cachar College, Silchar, Assam. Email: merin_in@yahoo.com. 
to dispense with the male bias of ethics is to accommodate women's experiences in it. It is a truism that non-inclusion of the variety of women's experiences stigmatized traditional ethics. Inclusion of the same is, therefore, a necessity. However, it needs to be ascertained in this context, that there is a sharp contrast in the approaches of mainstream ethics and feminist ethics towards the phenomenon of gender differences or gender distinctions. While gender distinctions served as the base for both exclusion of women's issues and sustenance of discriminatory practices in traditional ethics, feminist ethics wants to bring necessary correction in the same on the ground of inclusion of this difference only.

Question arises - if ethics cannot ignore or sideline the issue of gender distinctions or differences, how can it safeguard itself from the charge of perpetuating discriminations? While futility of the standards of universality and objectivity in gender blind ethics had been a matter of Feminists' common experience, introduction of gender and / or gender differences also equally created enough grounds for debates and controversies among various Feminists group. Differences of opinions are there as to the question which would be suitable goal for Feminist Ethics - Gender Neutrality or Gender Equality? Proponents of 'neutrality' thesis, like Susan Moller Okin, visualize a gender less society preconditioned by a massive restructuring of social institutions. But actualization of this symmetrist strategy sounds highly unrealistic at this stage of widespread prevalence of gender discriminations and gender violence in society. While on other hand, if the asymimetrists' pledge for equality is treated as worth considering, even then it is not risk free. Because an unreflective and simplistic approach towards the goal may frustrate the entire strategy. In either of the ways the issue of gender difference or distinction gets centrality in all the debates.

\section{The Concepts of Gender Neutrality and Gender Egalitarianism}

The ideal of "Gender Neutrality" speaks for a common human space beyond genders. Gender neutral analysis accordingly assumes that there is neutral impact on women and men of all 
social policy and programmes. Application of the same in the realm of ethics leads to the view that moral maxims or principles also do have neutral impact upon men and women irrespective of their gendered positionalities. Removal of specific male or female attributes very often becomes the precondition to this gender neutrality where consideration of gendered positions becomes redundant. This ideal situation is almost inconceivable in a social order where men and women do not yet enjoy equal rights and liberties. Obviously, Gender Equality becomes the prime necessity of the time.

Gender egalitarianism implies equality between men and women, which entails the concept that all human beings, both men and women, are free to develop their personal abilities and make choices without the limitations set by stereotypes, rigid gender roles and prejudices. Gender equality strives for equal consideration of the different behaviours, aspirations and needs of women and men that these are to be valued and favoured equally. It does not mean that women and men have to become the same; but that their rights, responsibilities and opportunities will not depend on the fact whether they are born male or female. Gender equity stands for fairness of treatment for women and men, according to their respective needs. This may include equal treatment or treatment that is different but which is considered equivalent in terms of rights, benefits, obligations and opportunities. That is to say women and men have equal conditions for realizing their full human rights and potential to contribute to national, political, economic, social and moral development and benefit equally from the results. Equality is essential for human development and peace. Aspiring for gender equality gives recognition to the fact that current social, economic, cultural, and political systems are gendered; those of women's unequal status is systemic hence, should be eliminated.

Gender equality requires equal enjoyment by women and men of socially valued goods, moral opportunities, resources and rewards. However, the emphasis on gender equality does not presume a particular model of gender equality for all societies and cultures, but reflects a concern that women and men have equal opportunities to make choices and work in partnership to achieve 
it. Because of current disparities, equal treatment of women and men is insufficient as a strategy for gender equality. Achieving gender equality will require changes in institutional practices and social relations through which disparities are reinforced and sustained. It also requires strengthening of women's voices in shaping of the societies. Empowerment of women is a necessary precondition to it. Equality between women and men should be promoted in ways that are appropriate to each in particular context. This begins with an understanding of the current status of women and men, their relations to each other and the interconnections between gender and other factors. Gender analysis offers an essential dimension and requisite methods to understand and address the socio-cultural reality. Gender analysis also makes it realized that there is an urgency to understand the social relations between men and women and the interconnection of gender with other social relationships, such as those defined by race, ethnicity, culture, class, age, disability and/or others status. Gender equity is the process of being fair to women and men. To ensure fairness, measures must often be available to compensate for historical and social disadvantages that prevent women and men from otherwise operating on level of parity. Equity leads to equality.

The feminist principle of equality goes at par with the phenomenon of inclusion. It reflects the need to address discrimination, prejudice and exclusion by including women and other marginalized groups in shaping the policies, programs, and practices, services and legislation that affect our lives. Inclusion is a process to bring about equality. It embraces individuals and groups who have been excluded from planning, decision-making and policy development within community. Inclusion empowers those, who have been traditionally excluded by providing opportunities, resources and support needed to participate. Feminist inclusion lens offer a way in which we can look at the root causes of long standing problems like sexism and other forms of discrimination, and consider new ways of thinking to solve these problems. Adoption of egalitarian approaches gives recognition to the importance of the two sexes (male and female) as complementary biological entities. Respecting the dignity and worth of the lives of people (of both genders) equally would be the goal of egalitarianism in any form. 
Eighteenth and nineteenth century Feminist philosophers like Wollstonecraft's and Mill's critique of Western Ethics is grounded on the fact that in it women's interests were never duly weighed like men. Hence, they demanded equal status for both men and women as regards their rights and privileges of. Of late it is an experience of the feminists that demand for identity of treatment for both men and women under gender blind laws gives the concept of sex / gender equality a formal colour. Formal equality does not necessarily result in substantive equality. Many of the Feminists' works in practical ethics have proved it beyond doubt that formally gender blind policies and practices very often are not gender neutral in their outcomes. To the contrary they have a disproportionately negative impact on women. Women are the worst sufferers in every context of scarcity, malnutrition, hunger, poverty, social evils, wars, environmental degradation, so on and so forth. It has been noticed that gender blind policies instead of reducing gender gaps are intensifying them more. In view of these, feminists have realized that construing sexual/gender equality in purely formal term may be inadequate for reaching substantive sexual or gender equality. It is more than an established truth that norms of gender situate women differently from men in most social contexts across the world. Achieving substantive equality may, therefore, necessitate establishing policies and practices that are gender-sensitive or gender responsive rather than gender blind.

While gender sensitivity is an urgent requirement in the sphere of ethics yet adjusting it with gender distinctions / differences may not be an easy task. The decade followed by 1980s had shown a significant turn in feminists' perception of equality - difference (or distinction) issue. Equality agenda constituted the principal goal of second-wave feminism since 1960. But, of late some feminists also have started developing critique of equality. The pledge of the asymmetrists (special rights pleaders) created a tension in the field. Because inclusion of 'differences' gradually landed into the plea for special rights for women in the matter of pregnancy, maternity or women's sexuality. This special rights issue was also impacting negatively people's perception of women's participatory roles. Less 
reliability upon women as workers due to their pre-occupations with pregnancy was supposed to perpetuate the same cultural myth about women's incompetence or fragility. Moreover, in some corners this apprehension was developed that gender responsive interpretations of sexual equality might not only provoke an antifeminist backlash, they might even undermine the prospect of long term sexual equality. In addition to that gender-responsive conceptions of equality were intended to reflect sensibly to differences in the circumstances of men and women in general and also to differences in the social situations of different women.

The Feminist agenda for equality must take a note of not only the internal differences which are systemic but also individual. It is necessary to recognize therefore, if providing child care to mothers in paid employment is a necessity, other mother's claims to welfare support is no less important an issue than the former. The need for special consideration in certain situation and in other a general one opens up two different areas of special rights and equal rights issue. These two kinds of rights, equal and differential (or special) work very differently. With regard to an equal right, taking a person's individual qualities into account may constitute discriminations. But with the special rights, they must be taken into account, for these rights are based on human differences. The special rights perspective was a contribution to Feminist legal thinking by Elizabeth Wolgast in 1980. According to her on installation of a system of special rights alongside equal rights women can best make their distinctive contribution to the culture and the society.

Plenty of Feminists, specially the legal practitioners in America of both Symmetrists (equal rights pleaders) and Asymmetrists (special rights pleaders) groups have experienced the tension in between equality - differences dilemmas. But by now it is clear that Feminist Ethics cannot be non-responsive either to gender differences or to gender equality. What it needs now is an adequate theoretical framework to accommodate both together. That is to say, the debates finally end with the necessity of theorizing an 'equality that 
acknowledges difference'. ${ }^{1}$ The core question is how to recognize notions of sexual or gender differences and yet to make an argument for equality in ethics. However, by subsuming women into general "human" diversity one cannot forget the specificity of female diversity and women experiences. Keeping this gender difference in mind we can claim for gender egalitarianism and also argue for a common space for both sexes on the basis of concrete universal norms, to be formulated not simply in terms of what is the same but in terms of the relevant differences also. As is said by Carol, C. Gould, "On the concrete view, the universal cannot be abstracted or conceived apart from their differences but exists only in and through them."2

The essentialists' consideration of sex difference led them to treat male and female as fixed natures apart from any particular social and historical context. But the notion of concrete universality can accommodate all those differences as that which emerged historically along with concrete forms of social interaction between men and women. So universal norms are constituted by an explicit specification of difference. The criterion of concrete universality presents a different picture of universal norms on the basis of which equality can be constituted on the ground of how individual is related to others and taking into account their specific difference. This is not to subscribe either to the view of abstract human universal or to any consideration of males and females as belonging to fundamentally different categories. The theoretical framework provided by the poststructuralist feminism also can be significant use here in understanding of this situation. A feminist poststructuralist approach not only addresses feminist theoretical debates about identity, equality and difference, but also opens up new opportunities for practitioners in managing diversity and

Lise Vogel, Women Questions, Essay for a Materialist Feminism, London: Pluto Press, 1995, 111-27.

2 C. Gould Carol, ed., The Women Question: Philosophy of Liberation and the Liberation of Philosophy. In Key concepts in Critical Theory of Gender, New Jersey: Humanities Press, 1997, 217. 
equality, to reflect on new dimensions of equality. Poststructuralism breaks the conceptual hold of long traditions of western philosophy that has systematically construed the world in terms of muscular universals and feminine specificity. The coexistence of equality and difference no longer presents any structurally impossible choice, as is argued by Scott. ${ }^{3}$ That is to say, sameness is not the sole ground on which equality can be pursued. Towards a Gender Egalitarian Ethics

\section{Reconciling Ethics of Care and Justice}

The dissolution of equality-difference or universality-specificity dichotomies is implicated in a fresh understanding of care-justice dichotomy too. The ethic of care, and especially the way in which it relates to theories of justice, has been one of the major streams in feminist ethics and political theory over the last decades. When the ethics of care started to take shape a few decades ago, care was generally seen as a value - or a set of values - opposed to justice. Care, presented by some as a feminist value and by others as a merely feminine one, is associated with compassion, emotional involvement, interest in one's particular circumstances and responsiveness to concrete needs. The values traditionally associated with justice - like impartiality, equality of resources and opportunity, fairness and focus on institutional structure - seemed to contradict the partiality of care and its emphasis on private relationships. Justice requires us to treat everybody according to the same standards, to treat friends in the same way as strangers, while an ethics of care praises mostly those acts done out of love and commitment for those who are near and dear. How could the two values be reconciled, then? Feminists pay close attention to care as a necessary condition for a just society. They argued that the more attention is paid to care as a precondition for social life, more it becomes obvious that care is a primary social good, whose

3 Joan W. Scott, “Deconstructing Equality versus Differences: Or, The Uses of Poststructuralist Theory for Feminism," in A.C. Hermann \& A.J. Stewart, ed., Theorizing Feminism, .S.A: Westview Press, 1994, 358-371. 
distribution is a matter of justice. Care is a necessary ingredient for the survival of any political community.

Recent advocates of an ethic of care acknowledge not only that some men think in terms of justice and women in terms of care, but also that most people of each sex are able to adopt either perspective. The "care orientation" focuses upon emotional relationships of attachment and networks of concrete relationships, connections, loyalties, and circles of concern whereas the "justice orientation" focuses upon equality, impartiality, universality, rules, and rights. While agents operating from the care orientation view human beings as so interdependent as to blur the boundaries demarcating them from one another, agents operating from the justice orientation are obsessed with the individual's autonomy and inclined to think of human beings in the most abstract way possible.

An ethic of care and responsibility develops from an individual's feeling of interconnectedness with others. It is contextual and arises from experience. It is characterized by nurturance and an emphasis on responsibilities to others. An ethic of justice, on the other hand, is an expression of autonomy. It is formulated in terms of universal, abstract principles and is characterized by rationality and an emphasis on individual rights. Some describe an ethic of caring as a "female" approach to morality and an ethic of rights and justice as a "male" approach. There are grounds which justify that the ethic of justice and the ethic of care be used complementary to each other for effective ethical decision making. It seems that the basic orientations of each conception are fundamentally different: the ethic of justice requires separation, while the ethic of care requires attachment. Separation is needed for the development and exercise of impartiality which, in turn, enables one to adjudicate moral conflict fairly. Attachment, on the other hand, is necessary for care, for care is based on empathy, and empathy is the recognition of interconnectedness. In other words, the ethic of care is founded upon reversibility. Without the capacity of reversibility it would be impossible to care. As discussed above, the ethic of justice also entails reversibility. 
Taking a clue from the popular view of care vs. justice orientations it may be argued so: if women care "better" than men, it may be epistemically, ethically and politically imprudent to dissociate women from or associate men with the virtue of care. Linking women and caring promoted the view that because women care, they should do so even at the cost of their own well-being. Whereas Gilligan has clearly made it a point to show how care, if used as a psychological law in a patriarchal social order, leads to serious subordination of women. Patriarchy reconciles the virtue of abnegation and care in women's morality satisfactorily. To the contrary consideration of care as a universal moral injunction is the demand of the ethic of care, which is sensitive to the gendered positioning as well. Gilligan's work plays a significant role here in showing how ethic of care dispenses with the limitations of psychology of care. However an unreflective understanding of care and justice perspectives may lead one to subscribe to the view that there can hardly be any human experience as much but only 'male experience' and 'female experience'. And also that highlighting the differences between men's and women's situations in life, both biologically and socially, makes one incapable of being reflective of universal human nature.

Some questions still remain: to what extent is either of these moral 'voices' (care and justice) related to gender? Can the ethic of care based on relatedness and responsiveness to others be considered a truly feminist ethic? What if we add in a principle or principles of feminism, the perspective of care? Can it be a principle of ethical deliberation, even accepting the naturalistic perspective that challenges all traditional values (justice, autonomy) as universal in character? Even if we accept such principles as justice as an ethical ideal and if it can be a principle, can it be triumphing principle, one that supersede other principles in a given context? If we treat moral orientations of justice and care as dichotomous then how can we claim for universal valid ethics for both sexes? Or no antagonism needs to be presumed between the two? Can both males and females do moral reasoning based on both justice and care? Can we ever come up with a design of justice - care blending and talk about multiplicity of moral perspectives among women as well as men? Should we subscribe to the view that gender distinctions may well be significantly spoken of and the context would still be 
moral? How can the ethic of justice and the ethic of care be used complementary to each other in ethical decision making? Liberal justice theorists tend to relegate care to the personal sphere and thereby conceptualize it as a form of self-interested action. Care theorists, like Gilligan, have recognized that the language of care tends to be ignored by justice theorists. But how far is it correct to identify justice and care as two genderized moralities?

Rawls' arguments in A Theory of Justice, presuppose care. Liberal justice theory should incorporate its own dependence on the 'ethic of care' into its theory, and work toward a conception of justice and care as reciprocal and interdependent. So there is a third way to view justice and care as complementary dimensions of morality. This complimentarity is, in turn, based upon an enlarged sense of self-identification from the personal to the ontological to the ecological. It may be proposed that this sequence of enlarged identification constitutes the development of morality as an integration of justice and care. On the highest level of abstraction the ethic of justice as fairness maintains that there exists a universal obligation to humanity. This obligation is generally conceived in terms of a universal commitment of respect for the inherent dignity of all persons. The ethic of care, on the other hand, maintains that moral obligation is grounded in the particulars of the moral context. In this sense the ethic of care grounds morality in an ethic of association. The ethic of association maintains that moral responsibility is based on particular human relationships. Ethical obligation emerges out of relationship, and it is care, from the feminist perspective, that is the central emergent property of ethical association. However, while perceiving the associative quality of morality as essential, the ethic of care is also based upon a universal commitment to our shared humanity. Gilligan herself concludes that the essential motivation of an ethic of care is "that everyone will be responded to and included, that no one will be left alone or hurt." 4 These statements suggest that a commitment to our shared humanity in a universal sense, rather than one that is founded upon particular and immediate association is as basic to the ethic of Theory, New York: Oxford University Press, 1990, 271. 
care as it is to an ethic of justice. The web of relations extends beyond immediate association to include an ontological interdependence.

Responsibility is essentially a function of one's capacity to respond to others, whether in terms of their needs (care) or in terms of respect (fairness). ${ }^{5}$ Being responsive is the ontological foundation of moral obligation and, hence, moral development. And, responsiveness is a function of identification. Responsiveness occurs within a relational field. It requires and is constituted by relationship. Responsible relationship implies a mutually permissive interaction that can only occur if there is resonance between the individuals who are in relationship. Resonance, in turn, occurs on the basis of identification. Therefore, if there is no identification, there will be no response and, hence, neither care nor fairness. As one's identification expands or contracts, one's capacity to respond and, thus, to act morally, expands or contracts. This is the essence of reversibility as a moral foundation, and it applies to both care and justice.

Care entails the embracing of interconnectedness. The expansion of identification is one of unification with the other -- recognizing the web of relationships that literally connect our identities. Whereas the expansion of identification entailed in justice does not imply interconnection per se, but it is an identification that recognizes the sameness of interpersonal boundaries that define individuality within a relational field. This identification of the boundaries of others as identical to the boundaries that define our own individuality is the basis of respect. This kind of identification is recognition of the fact that, just as one does not want one's private boundaries violated, neither do others. This accounts for justice as respect. This notion of responsibility is essentially negative in that it requires a response that prohibits interference (the essence of respect for boundaries), whereas the ethic of care entails a positive Ethics. New York: Henry Holt and Company, 1947, 234. 
conception of responsibility in that it requires a response to help, to provide in order to satisfy a need. Eco-philosophies, such as Deep Ecology, Social Ecology and Eco-feminism, are all possible contenders for such a philosophy in that they clearly articulate such concepts as interrelatedness, oneness, wholeness, completeness and inter-connectedness of human beings with everything around them.

\section{Conclusion}

The complementarity between justice and care is expressed in the ecosophical conception of morality put forth by Arne Naess. ${ }^{6}$ The justice and care orientations of Kohlberg and Gilligan have important features in common which relate directly to Naess's ecosophy. Morality is not conceived as an abstract body of rules, but as a state of being. Morality here is not a body of principles to be obeyed, but a way of being in the world, in relation to other human beings as well as nature. From an ecosophical perspective, we exist in a web of relations that extends to nature itself. Naess's view is consistent with the ethic of care, even extending the sphere of association to include not only the web of human relations, but the ecological web. However, we do not exist with complete autonomy: we are fundamentally related and simultaneously individuated. Just as our interrelatedness requires care, our individuated status requires respect. Naess states it this way: "In the thinking of the field ecologist there is respect for an extreme diversity of beings capable of living together in an intricate web of relations." 7

When we care for and respect another being or even the earth, we are engaged in an act of friendship. ${ }^{8}$ Friendship is a relationship of love which allows the other person to be authentic. In facilitating

6 Arne Naess, Ecology, Community and Life style, trans. \& ed., David Rothenberg, New York: Cambridge University Press, 1989, 132

7 Arne Naess, Ecology, Community and Life style, 257.

8 Warwick Fox, Toward a Transpersonal Ecology, Boston: Shambala Press, 1990, 217. 
the authenticity of the other, we are simultaneously caring for and respecting them, acknowledging and nurturing our interdependence while simultaneously affirming our individual uniqueness. Friendship, in this sense, is the organizing catalyst for the expansion of self and the proportional expansion of our moral capacity for care and fairness. The growth of friendship generally proceeds in terms of personal interaction and identification. We become friends and treat others with care and respect those who we have come to know and appreciate personally. However, friendship and, thus, care and respect can proceed in terms of ontological and even ecological identification. ${ }^{9}$ We can befriend nature and the earth by expanding our sense of self through a recognition of ecological interdependence. Here our identification is ecological and, in turn, our moral sensibility enlarges to include the natural environment.

9 Warwick Fox, Toward a Transpersonal Ecology, Ch. 8. 